Authentizität und Repräsentativität des Datenmaterials. Dabei sollte diese Diskussion doch wohl am Anfang jeder computerlinguistischen Erhebung stehen.

Am Ende eine ganz laienhaft computerlinguistisch ermittelte Information zum Fachsprachgebrauch von Linguisten. Was halten Sie für frequenter: "gesprochensprachlich « (Hundt, 29) oder »sprechsprachlich « (Kaufmann, 75)?

Antwort: Ende Oktober 2008 ergibt "gesprochensprachlich « 247, "sprechsprachlich« 3.790 Googles. Die Frage ist, und damit sind wir am Anfang der Computerlinguistik, welche Konsequenzen Frequenzen haben. Es wird ohnehin der Tag kommen, an dem wir aufgrund von ins Uferlose gehenden quantitativen Erhebungen hin und wieder >lexikalischen (und auch grammatischen) Minderheitenschutz \& fordern und darauf bestehen werden, so zu reden und $\mathrm{zu}$ schreiben, wie es computerlinguistisch für gar nicht möglich gehalten werden wird.

\section{Li, Yuan:}

Integrative Landeskunde. Ein didaktisches Konzept für Deutsch als Fremdsprache in China am Beispiel des Einsatzes von Werbung. München: iudicium, 2007. - ISBN 978-3-89129-587-8. 288

Seiten, $€ 30,00$

\section{(Conny Bast, Straßberg)}

Die meisten Fremdsprachenlehrer kennen das Problem: Unterrichtet man Landeskunde innerhalb des Sprachunterrichts anhand von authentischen Texten, sind die Themen (besonders im Anfängerunterricht) insgesamt beschränkt. Unterrichtet man faktische Landeskunde als eigenständiges Fach, erhalten die Studenten zwar viel theoretisches Wissen zur Zielkultur, es fehlt jedoch häufig der Bezug zur Praxis und zum späteren
Beruf. Aus diesem Grund stellt Yuan Li in der hier vorliegenden Dissertation das von ihr entwickelte Konzept der integrativen Landeskunde vor, welches sie am Beispiel des Einsatzes von Werbung in China erklärt. Das Buch besteht aus 6 Kapiteln nebst einer Einleitung, einem Anhang, der die besprochene Werbung zeigt, und einem Literaturverzeichnis, welches in Literatur auf Deutsch und Literatur auf Chinesisch unterteilt ist.

Kapitel 1 beginnt mit der Darstellung der Didaktik der Landeskunde in Deutschland und gibt zunächst einen Überblick über die Probleme der Benennung und der Definition des Begriffs Landeskunde (19). Li beschreibt anschaulich, dass sich die Landeskunde in drei Blöcken entwickelt hat. Sie galt zunächst als Kulturkunde zur Geistesbildung und sollte den Beweis der Überlegenheit des deutschen Wesens liefern (26), ein Ansatz, der im Dritten Reich von den Nationalsozialisten zu Propagandazwecken missbraucht wurde. Ein weiterer Ansatz ist die Landeskunde als Realienkunde. Sie sollte Informationen über andere Nationen liefern, um auf die Globalisierung bzw. den Konkurrenzkampf vorzubereiten. Seit den 60er Jahren gibt es den faktenorientierten Ansatz, bei dem enzyklopädisches Wissen vermittelt wird, die kommunikative Landeskunde im Dienst des sprachlichen Handelns und den interkulturellen Ansatz zum Verstehen des Fremden und des Eigenen. Jeder Ansatz wird beschrieben und kritisch beleuchtet. In Kapitel 2 stellt Li dar, wie sich das Herangehen an Landeskunde in China von dem in Deutschland unterscheidet. Bis zum Ende der 80er Jahre kam es in erster Linie auf die Beherrschung von Wortschatz und Grammatik und nicht auf das Wissen über das jeweilige Land an. Bis heute ist der faktenorientierte Ansatz vorherrschend und starken politischen Erwartungen unterworfen (70). Be- 
tont werden kulturelle Differenzen, und nicht amwendbares Wissen, sondern Informationen und Daten bilden den Gegenstand der Landeskunde. Unterrichtet wird im Frontalunterricht mit engen Themenbereichen, es erfolgt keine Lernerorientierung und der Unterricht ist nicht problemorientiert, eine Herangehensweise, die leider nicht nur auf China beschränkt ist.

Um hier Abhilfe zu schaffen, entwickelte Li das Konzept der integrativen Landeskunde. Dies geschah im Kontext von German Studies an der Zhejiang Universität in Hangzhou, VR China, an der sie als Professorin tätig ist. Im 3. Kapitel stellt sie nun die konkrete Situation in ihrem Fachbereich vor. Sie stellt fest, dass es ihren Studierenden an der bewussten Reflexion über eigene Erfahrungen und Erlebnisse in der interkulturellen Begegnung mangelt (100). Außerdem fehlt es ihren Lernergruppen an Motivation, aktiver Teilnahme und an Lern- und Arbeitstechniken zur Bewältigung späterer beruflicher Anforderungen. Daher strebt sie danach, in integrativer Landeskunde Fachwissen mit Alltagssituationen und interkulturellem Ansatz so zu kombinieren, wie es den Bedürfnissen der Studierenden entspricht. Integrative Landeskunde wird »explizit zum Lerngegenstand gemacht « (107) und das Sprachlernen wird in den Landeskunde-Unterricht integriert. Im Vordergrund stehen Handlungskompetenzen im interkulturellen Kontext. Zusätzlich zu diesen Kompetenzen lernen die chinesischen Studierenden Methoden zur Wissensaneignung kennen, die selbständiges Denken, Abstraktionsvermögen, Analyse- und Kritikfähigkeit erfordern, ein bisher noch recht unbekanntes Konzept in China.

Bevor nun konkret auf die Anwendung von Werbung eingegangen wird, erfährt der Leser in Kapitel 4 mehr als ausführlich, was man unter dem Begriff Werbung überhaupt versteht, wie Werbung unter der Kommunikationsperspektive gesehen wird und dass manche Werbungen in China und Deutschland identisch sind.

Kapitel 5 beinhaltet den empirischen Teil der Untersuchung, der sich auf teilnehmende und nicht-teilnehmende Beobachtungen sowie auf Befragungen zum integrativen Landeskunde-Unterricht stützt. Als Unterrichtsgegenstand verwendet $\mathrm{Li}$ eine chinabezogene Werbung einer deutschen Bank. Die Werbung wird zunächst ausführlich dargestellt, danach geht Li auf das Chinabild und das Bankwesen in Deutschland ein. Dies dient dazu, den chinesischen Studierenden die Interdependenz der Kulturen und die Auseinandersetzung mit dem Eigenbild und dem Fremdbild näherzubringen, und es zeigt ihnen auch anschaulich, dass man mit Stereotypen sehr kritisch umgehen muss. Nach Aussage der befragten Studierenden war dieser neue Ansatz für sie sehr interessant und erheblich ansprechender als die Vermittlung trockener Fakten ohne persönlichen Bezug.

In Kapitel 6 schlussfolgert Li, wie ein erfolgreiches Curriculum der integrativen Landeskunde am Beispiel des Einsatzes von Werbung aussehen kann. Sie fasst noch einmal die Rahmenbedingungen zusammen, nennt die zu beachtenden Komponenten integrativer Landeskunde sowie die Kriterien zur Auswahl der Werbung. Weiterhin geht sie auf die Methoden, die zu verwendenden Medien und die Evaluationsformen ein.

Insgesamt hätte ich mir (besonders im empirischen Teil) eine wesentlich ausführlichere, praktische Beschreibung zum Einsatz der Werbung im Bereich Landeskunde gewünscht. Das empirische Kapitel mit seinen nur 25 Seiten ist definitiv zu kurz. Dennoch geben die hier vermittelten Ideen viele interessante Vorschläge für den Landeskunde-Unterricht im Ausland. 